\title{
INTERNAL HEAT SOURCE IN THERMOELASTIC MICROELONGATED SOLID UNDER GREEN LINDSAY THEORY*
}

\author{
Praveen Ailawalia \\ Department of Applied Sciences and Humanities, \\ Maharishi Markandeshwar University, Sadopur, Ambala, Haryana, India, \\ e-mail : praveen_2117@rediffmail.com \\ Sunil Kumar Sachdeva \\ Department of Applied Sciences, D.A.V Institute of Engineering and Technology, \\ Jalandhar, Punjab, India \\ e-mail: sunilsachdeva.daviet@gmail.com \\ and \\ Research Scholar, IKG Punjab Technical University, \\ Jalandhar, Punjab, India \\ Devinder Singh Pathania \\ Department of Applied Sciences, Guru Nanak Dev Engineering College, \\ Ludhiana, Punjab, India \\ [Received 25 February. Accepted 20 June 2016]

\begin{abstract}
The present study deals with two dimensional deformation, due to internal heat source in a thermoelastic microelongated solid. A mechanical force is applied along the interface of elastic half space and thermoelastic microelongated half space. The problem is in the context of Green Lindsay (GL) theory. The analytic expressions for displacement component, normal force stress, temperature distribution and microelongation have been derived. The effect of internal heat source and microelongation on the derived components have been depicted graphically.

KEY WORDS: Thermoelasticity, microelongation, heat source, normal mode, elastic solid.
\end{abstract}

\section{Introduction}

Classical elasticity is inadequate to discuss the behaviour of materials possessing internal structure. The micropolar elastic model is more realistic than the purely elastic theory to study the response of materials to external stimuli. Eringen and Suhubi [1-2] developed a non linear theory of micro elastic

\footnotetext{
${ }^{*}$ Corresponding author e-mail: praveen_2117@rediffmail.com.
} 
solids. Later on, a theory was framed in which material particles in solids can undergo macro-deformations, as well as micro-rotations by Eringen [3-5] and named the theory as "linear theory of micropolar elasticity".

The axial stretch was included during the rotation of molecules by Eringen [6] and named the theory as micropolar elastic solid with stretch. Nowacki [7], Eringen [8], Tauchert et al. [9], Tauchert [10] and Nowacki and Olszak [11] included thermal effects in the micropolar theory. One can refer to Dhaliwal and Singh [12] for a review on the micropolar thermoelasticity and a historical survey of the subject. The general theory of micromorphic media has been summed up in "Continuum Physics" series by Eringen and Kafadar [13]. Lord and Shulman [14] and coupled theory of elasticity are two important generalized theories of thermoelasticity. Entropy production inequality was proposed by Muller [15]. A generalization of this inequality was proposed by Green and Laws [16]. Green and Lindsay [17] obtained another version of these constitutive equations. These equations were also obtained independently and more explicitly by Suhubi [18]. This theory contains two constants that act as relaxation times and modify all the equations of coupled theory, not only the heat equations. The classical Fourier law of heat conduction is not violated, if the medium under consideration has a centre of symmetry.

Barber [19] studied thermoelastic displacements and stresses due to a heat source moving over the surface of a half plane. Sherief [20] obtained components of stress and temperature distributions in a thermoelastic medium due to a continuous source. Dhaliwal et al. [21] investigated thermoelastic interactions, caused by a continuous line heat source in a homogeneous isotropic unbounded solid. Chandrasekharaiah and Srinath [22] studied thermoelastic interactions due to a continuous point heat source in a homogeneous and isotropic unbounded body. Sharma et al. [23] investigated the disturbance due to a time-harmonic normal point load in a homogeneous isotropic thermoelastic half-space. Sharma and Chauhan [24] discussed mechanical and thermal sources in a generalized thermoelastic half-space. Sarbani and Amitava [25] studied the transient disturbance in a half-space due to moving internal heat source under Lord-Shulman model. Youssef [26] found the solution for the problem on a generalized thermoelastic infinite medium with a spherical cavity, subjected to a moving heat source.

A microelongated elastic solid possesses four degrees of freedom: three for translation and microelongation. In microelongation theory, the material particles can perform only volumetric micro elongation in addition to classical deformation of the medium. The material points of such medium can stretch and contract independently of their translations. Solid liquid crystals, 
composite materials reinforced with chopped elastic fibres, porous media with pores filled with non-viscous fluid or gas can be categorized as microelongated medium. Shaw and Mukhopadhyay [27] discussed the variation of periodical heat source response in a functionally graded microelongated medium. Shaw and Mukhopadhyay [28] studied the thermoelastic interactions in a microelongated, isotropic, homogeneous medium in the presence of a moving heat source. Ailawalia and Sachdeva discussed plane strain problem in a thermoelastic microelongated solid with an overlying infinite non-viscous fluid [34].

In the present problem, deformation due to internal heat source in a thermoelastic microelongated solid along the interface of elastic half space has been discussed. The normal mode analysis is applied to derive the expressions for the considered variables for Green Lindsay (GL) theory of thermoelasticity and the variations of the considered variables are represented graphically.

\section{Problem formulation}

The constitutive equation for a homogeneous, isotropic, microelongated, thermoelastic solid are [28]:

$$
\begin{gathered}
\sigma_{k l}=\lambda \delta_{k l} u_{r, r}+\mu\left(u_{k, l}+u_{l, k}\right)-\beta_{0}\left(1+t_{1} \delta_{2 k} \frac{\partial}{\partial t}\right) T \delta_{k l}+\lambda_{0} \delta_{k l} \varphi \\
m_{k}=a_{0} \varphi_{, k}, \\
s-\sigma=\lambda_{0} u_{k, k}-\beta_{1}\left(1+t_{1} \delta_{2 k} \frac{\partial}{\partial t}\right) T+\lambda_{1} \varphi \\
q_{k}=\frac{K^{*}}{T_{0}} T_{, k},
\end{gathered}
$$

where, $\beta_{0}=(3 \lambda+2 \mu) \alpha_{t_{1}}, \beta_{1}=(3 \lambda+2 \mu) \alpha_{t_{2}}$.

The field equation of motion, according to $[29,30]$ and heat conduction equation according to [31] for the displacement, microelongation and temperature changes are:

$$
(\lambda+\mu) u_{j, i j}+\mu u_{i, j j}-\beta_{0}\left(1+t_{1} \delta_{2 k} \frac{\partial}{\partial t}\right) T_{, i}+\lambda_{0} \varphi_{, i}=\rho \ddot{u}_{i},
$$

$$
a_{0} \varphi_{, i i}+\beta_{1}\left(1+t_{1} \delta_{2 k} \frac{\partial}{\partial t}\right) T-\lambda_{1} \varphi-\lambda_{0} u_{j, j}=\frac{1}{2} \rho j_{0} \ddot{\varphi}
$$




$$
\begin{aligned}
K^{*} T_{, i i}-\rho C^{*}\left(1+t_{0} \delta_{1 k} \frac{\partial}{\partial t}\right) \dot{T}-\beta_{0} T_{0} & \left(1+t_{0} \delta_{1 k} \frac{\partial}{\partial t}\right) \dot{u}_{k, k} \\
& \quad-\beta_{1} T_{0} \dot{\varphi}+\rho\left(1+t_{0} \delta_{1 k} \frac{\partial}{\partial t}\right) Q=0 .
\end{aligned}
$$

The equation of motion and stress components in an elastic medium are given by [32]:

$$
\begin{gathered}
\left(\lambda^{e}+\mu^{e}\right) u_{j, i j}^{e}+\mu^{e} u_{, i i}^{e}=\rho^{e} \ddot{u}_{i}^{e}, \\
\sigma_{i j}^{e}=\lambda^{e} u_{j, j}^{e} \delta_{i j}+\mu^{e}\left(u_{i, j}^{e}+u_{j, i}^{e}\right) .
\end{gathered}
$$

We consider a normal force of magnitude $P_{1}$, acting along the interface of microelongated thermoelastic solid half space, occupying the region $0 \leq x \leq$ $\infty$ and an elastic solid half space in the region $-\infty \leq x \leq 0$, as shown in Fig. 1. We have restricted our analysis to the plane strain parallel to $x y$ plane with displacement vector:

$$
\vec{u}_{i}=\left(u_{1}, u_{2}, 0\right) \text { and } \vec{u}_{i}^{e}=\left(u_{1}^{e}, u_{2}^{e}, 0\right) .
$$

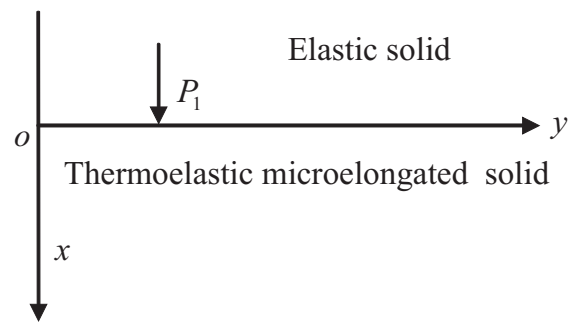

Fig. 1. Geometry of the problem

To simplify calculations, we use following non-dimensional variables:

$$
\begin{gathered}
x^{\prime}=\frac{\omega^{*}}{c_{1}} x, \quad y^{\prime}=\frac{\omega^{*}}{c_{1}} y, \quad u_{i}^{\prime}=\frac{\omega^{*} \rho c_{1}}{\beta_{0} T_{0}} u_{i}, \quad u_{i}^{e^{\prime}}=\frac{\omega^{*} \rho c_{1}}{\beta_{0} T_{0}} u_{i}^{e}, \quad t^{\prime}=\omega^{*} t, \\
t_{0}^{\prime}=\omega^{*} t_{0}, \quad t_{1}^{\prime}=\omega^{*} t_{1}, \quad \sigma_{i j}^{\prime}=\frac{\sigma_{i j}}{\beta_{0} T_{0}}, \quad \varphi^{\prime}=\frac{\lambda_{0}}{\beta_{0} T_{0}} \varphi, \quad \sigma_{i j}^{e^{\prime}}=\frac{\sigma_{i j}^{e}}{\beta_{0} T_{0}}, \\
P_{1}^{\prime}=\frac{P_{1}}{\beta_{0} T_{0}}, \quad T^{\prime}=\frac{T}{T_{0}}, \quad Q^{\prime}=\frac{1}{\omega^{*} c_{1}^{2}} Q,
\end{gathered}
$$

where, $\omega^{*}=\frac{\rho c_{1}^{2} C^{*}}{K^{*}}, c_{1}^{2}=\frac{\lambda+2 \mu}{\rho}$. 
Internal Heat Source in Thermoelastic Microelongated Solid ...

Using above non dimensional variables and (9a) in equations (5)-(7), we obtain the following non dimensional equations (after dropping superscripts):

$$
l_{1} \frac{\partial^{2} u_{1}}{\partial x^{2}}+l_{2} \frac{\partial^{2} u_{2}}{\partial x \partial y}+l_{3} \frac{\partial^{2} u_{1}}{\partial y^{2}}-\left(1+t_{1} \delta_{2 k} \frac{\partial}{\partial t}\right) \frac{\partial T}{\partial x}+\frac{\partial \varphi}{\partial x}=\frac{\partial^{2} u_{1}}{\partial t^{2}},
$$

$$
l_{3} \frac{\partial^{2} u_{2}}{\partial x^{2}}+l_{2} \frac{\partial^{2} u_{1}}{\partial x \partial y}+l_{1} \frac{\partial^{2} u_{2}}{\partial y^{2}}-\left(1+t_{1} \delta_{2 k} \frac{\partial}{\partial t}\right) \frac{\partial T}{\partial y}+\frac{\partial \varphi}{\partial y}=\frac{\partial^{2} u_{2}}{\partial t^{2}},
$$

$$
\begin{aligned}
& \left(\frac{\partial^{2} \varphi}{\partial x^{2}}+\frac{\partial^{2} \varphi}{\partial y^{2}}\right)+l_{4}\left(1+t_{1} \delta_{2 k} \frac{\partial}{\partial t}\right) T-l_{5} \varphi-l_{6}\left(\frac{\partial u_{1}}{\partial x}+\frac{\partial u_{2}}{\partial y}\right)=l_{7} \frac{\partial^{2} \varphi}{\partial t^{2}} \\
& \left(\frac{\partial^{2} T}{\partial x^{2}}+\frac{\partial^{2} T}{\partial y^{2}}\right)-l_{8}\left(1+t_{0} \delta_{1 k} \frac{\partial}{\partial t}\right) \frac{\partial T}{\partial t} \\
& -l_{9}\left(\frac{\partial}{\partial t}+t_{0} \delta_{1 k} \frac{\partial^{2}}{\partial t^{2}}\right)\left(\frac{\partial u_{1}}{\partial x}+\frac{\partial u_{2}}{\partial y}\right)-l_{10} \frac{\partial \varphi}{\partial t}+l_{11}\left(1+t_{0} \delta_{1 k} \frac{\partial}{\partial t}\right) Q=0
\end{aligned}
$$

The dimensionless constitutive relations are:

$$
\begin{gathered}
\sigma_{x x}=l_{1} \frac{\partial u_{1}}{\partial x}+l_{12} \frac{\partial u_{2}}{\partial y}-\left(1+t_{1} \delta_{2 k} \frac{\partial}{\partial t}\right) T+\varphi, \\
\sigma_{x y}=l_{3}\left(\frac{\partial u_{1}}{\partial y}+\frac{\partial u_{2}}{\partial x}\right), \\
\sigma_{y y}=l_{12} \frac{\partial u_{1}}{\partial x}+l_{1} \frac{\partial u_{2}}{\partial y}-\left(1+t_{1} \delta_{2 k} \frac{\partial}{\partial t}\right) T+\varphi
\end{gathered}
$$

where, the coefficients $l_{i}$ are given in Appendix-1.

\section{Normal mode analysis}

The solution of the considered physical variables can be decomposed in terms of normal mode as:

$$
\left(u_{i}, u_{i}^{e}, T, \varphi, \sigma_{i j}, \sigma_{i j}^{e}, Q\right)(x, y, t)=\left(u_{i}^{*}, u_{i}^{e^{*}}, T^{*}, \varphi^{*}, \sigma_{i j}^{*}, \sigma_{i j}^{e^{*}}, Q^{*}\right)(x) e^{\omega t+i b y},
$$

where, $u_{i}^{*}(x), u_{i}^{e^{*}}(x), T^{*}(x), \varphi^{*}(x), \sigma_{i j}^{*}(x), \sigma_{i j}^{e^{*}}(x), Q^{*}$ are the amplitudes of field quantities. 
Using normal mode in equation (10)-(13), we get:

$$
\begin{gathered}
\left(l_{1} D^{2}-B_{1}\right) u_{1}^{*}+i b l_{2} D u_{2}^{*}-B_{2} D T^{*}+D \varphi^{*}=0, \\
i b l_{2} D u_{1}^{*}+\left(l_{3} D^{2}-B_{3}\right) u_{2}^{*}-i b B_{2} T^{*}+i b \varphi^{*}=0, \\
-l_{6} D u_{1}^{*}-i b l_{6} u_{2}^{*}+B_{2} l_{4} T^{*}+\left(D^{2}-B_{4}\right) \varphi^{*}=0, \\
-l_{9} B_{6} D u_{1}^{*}-i b l_{9} B_{6} u_{2}^{*}+\left(D^{2}-B_{7}\right) T^{*}-l_{10} \omega \varphi^{*}=-l_{11} B_{5} Q^{*}, \\
\sigma_{x x}^{*}=l_{1} D u_{1}^{*}+i b l_{12} u_{2}^{*}-B_{2} T^{*}+\varphi^{*}, \\
\sigma_{y y}^{*}=l_{12} D u_{1}^{*}+i b l_{1} u_{2}^{*}-B_{2} T^{*}+\varphi^{*}, \\
\sigma_{x y}^{*}=l_{3}\left(i b u_{1}^{*}+D u_{2}^{*}\right),
\end{gathered}
$$

where, the coefficients $B_{j}$ are given in Appendix 2 .

Eliminating $u_{2}^{*}(x), T^{*}(x), \varphi^{*}(x)$ from equations (17)-(20), we get:

$$
\left(D^{8}+A D^{6}+B D^{4}+C D^{2}+E\right) u_{1}^{*}(x)=R Q^{*},
$$

where, the coefficients $A, B, C, E$ and $R$ are given in Appendix 3.

Similarly, $u_{2}^{*}(x), T^{*}(x), \varphi^{*}(x)$ satisfies the equation:

$$
\left(D^{8}+A D^{6}+B D^{4}+C D^{2}+E\right)\left(u_{2}^{*}(x), T^{*}(x), \varphi^{*}(x)\right)=R Q^{*},
$$

which can be written as:

$$
\left(D^{2}-k_{1}^{2}\right)\left(D^{2}-k_{2}^{2}\right)\left(D^{2}-k_{3}^{2}\right)\left(D^{2}-k_{4}^{2}\right) u_{1}^{*}(x)=R Q^{*},
$$

where $k_{n}^{2},(n=1,2,3,4)$ are the roots of characteristic equation (25).

The series solution for the physical quantities are given by:

$$
\begin{aligned}
& u_{1}^{*}(x)=\sum_{n=1}^{4}\left[M_{n}(b, \omega) e^{-k_{n} x}\right]+S, \\
& u_{2}^{*}(x)=\sum_{n=1}^{4}\left[M_{n}^{\prime}(b, \omega) e^{-k_{n} x}\right]-S_{1},
\end{aligned}
$$


Internal Heat Source in Thermoelastic Microelongated Solid ...

$$
\begin{aligned}
& T^{*}(x)=\sum_{n=1}^{4}\left[M_{n}^{\prime \prime}(b, \omega) e^{-k_{n} x}\right]-S_{2}, \\
& \varphi^{*}(x)=\sum_{n=1}^{4}\left[M_{n}^{\prime \prime \prime}(b, \omega) e^{-k_{n} x}\right]-S_{3},
\end{aligned}
$$

where, $M_{n}(b, \omega), M_{n}^{\prime}(b, \omega), M_{n}^{\prime \prime}(b, \omega), M_{n}^{\prime \prime \prime}(b, \omega)$ are specific functions, depending upon $b$ and $\omega$.

Using equation (27)-(30) in equation (17)-(20), we get:

$$
M_{n}^{\prime \prime \prime}(b, \omega)=H_{3 n} M_{n}(b, \omega) .
$$

Using (31)-(33), the series solution takes the form:

$$
\begin{aligned}
& u_{2}^{*}(x)=\sum_{n=1}^{4}\left[H_{1 n} M_{n}(b, \omega) e^{-k_{n} x}\right]-S_{1}, \\
& T^{*}(x)=\sum_{n=1}^{4}\left[H_{2 n} M_{n}(b, \omega) e^{-k_{n} x}\right]-S_{2},
\end{aligned}
$$

$$
\varphi^{*}(x)=\sum_{n=1}^{4}\left[H_{3 n} M_{n}(b, \omega) e^{-k_{n} x}\right]-S_{3},
$$

$$
\begin{aligned}
& \sigma_{x x}^{*}(x)=\sum_{n=1}^{4}\left[H_{4 n} M_{n}(b, \omega) e^{-k_{n} x}\right]+S_{4}, \\
& \sigma_{x y}^{*}(x)=\sum_{n=1}^{4}\left[H_{5 n} M_{n}(b, \omega) e^{-k_{n} x}\right]+S_{5},
\end{aligned}
$$




$$
\sigma_{y y}^{*}(x)=\sum_{n=1}^{4}\left[H_{6 n} M_{n}(b, \omega) e^{-k_{n} x}\right]+S_{6},
$$

where, coefficients $H_{i j}, S$ and $S_{j}$ are given in Appendix 4 .

Similarly, for the elastic half space, the solutions are of the form:

$$
u_{1}^{e^{*}}(x)=\sum_{n=1}^{2}\left[R_{n}(b, \omega) e^{r_{n} x}\right]
$$

$$
u_{2}^{e^{*}}(x)=\sum_{n=1}^{2}\left[R_{n}^{\prime}(b, \omega) e^{r_{n} x}\right],
$$

where $R_{n}(b, \omega)$ and $R_{n}^{\prime}(b, \omega)$ are specific functions, depending upon $b$ and $\omega$ and $r_{n}^{2},(n=1,2)$ are roots of the equation:

$$
\left(D^{4}-G D^{2}+L\right) u_{1}^{e^{*}}(x)=0 .
$$

And solutions of physical quantities are given by:

$$
u_{2}^{e^{*}}(x)=\sum_{n=1}^{2}\left[L_{1 n} R_{n}(b, \omega) e^{r_{n} x}\right]
$$

$$
\sigma_{x x}^{e^{*}}(x)=\sum_{n=1}^{2}\left[L_{2 n} R_{n}(b, \omega) e^{r_{n} x}\right]
$$

$$
\sigma_{y y}^{e^{*}}(x)=\sum_{n=1}^{2}\left[L_{3 n} R_{n}(b, \omega) e^{r_{n} x}\right]
$$

$$
\sigma_{x y}^{e^{*}}(x)=\sum_{n=1}^{2}\left[L_{4 n} R_{n}(b, \omega) e^{r_{n} x}\right],
$$

where, the coefficients $G, L$ and $L_{k j}$ are given in Appendix 5 . 


\section{Applications}

We suppress the positive exponentials in the physical problem to determine the parameters $M_{n},(n=1,2,3,4)$ and $R_{n},(n=1,2)$, which are unbounded at infinity. Constants $M_{1}, M_{2}, M_{3}, M_{4}$ and $R_{1}, R_{2}$ have to be selected, such that boundary conditions at the surface $x=0$ are:

$$
\sigma_{x x}=\sigma_{x x}^{e}-P_{1} e^{\omega t+i b y}, u_{1}=u_{1}^{e}, u_{2}=u_{2}^{e}, \sigma_{x y}=\sigma_{x y}^{e}, \varphi=0, \frac{\partial T}{\partial x}=0 .
$$

where $P_{1}$ is the magnitude of the mechanical force.

Using the expressions of $\sigma_{x x}, \sigma_{x x}^{e}, u_{1}, u_{1}^{e}, u_{2}, u_{2}^{e}, \sigma_{x y}, \sigma_{x y}^{e}, T, \varphi$ into above boundary conditions, we get:

$$
\begin{gathered}
\sum_{n=1}^{4}\left[H_{4 n} M_{n}\right]-\sum_{n=1}^{2}\left[L_{2 n} R_{n}\right]=-P_{1}-S_{4}, \quad \sum_{n=1}^{4}\left[M_{n}\right]-\sum_{n=1}^{2}\left[R_{n}\right]=-S, \\
\sum_{n=1}^{4}\left[H_{1 n} M_{n}\right]-\sum_{n=1}^{2}\left[L_{1 n} R_{n}\right]=-S_{1} \quad \sum_{n=1}^{4}\left[H_{5 n} M_{n}\right]-\sum_{n=1}^{2}\left[L_{4 n} R_{n}\right]=-S_{5}, \\
\sum_{n=1}^{4}\left[H_{3 n} M_{n}\right]=S_{3}, \quad \sum_{n=1}^{4}\left[H_{2 n} k_{n} M_{n}\right]=0 .
\end{gathered}
$$

After solving the above system of non homogeneous equations, we get the values of constant $M_{1}, M_{2}, M_{3}, M_{4}, R_{1}, R_{2}$ and hence, obtain the components of normal displacement, normal force stress, temperature distribution and microelongation at the interface of microelongated thermoelastic half space and elastic half space.

\section{Special Cases}

1. If we neglect microelongation effect i. e. $\lambda_{0}=\beta_{1}=\lambda_{1}=a_{0}=j_{0}=$ 0 , we obtain the results for thermoelastic solid (TS).

2. Letting $Q \rightarrow 0$ in (7) and $\mu^{e} \rightarrow 0$ in (8) and (9), problem reduces to a plane strain problem in thermoelastic microelongated solid with an overlying infinite non viscous fluid [34].

\section{Numerical results and discussions}

For numerical computations, we consider the values of constants for aluminium epoxy-like material as [28]:

$$
\lambda=7.59 \times 10^{10} \mathrm{~N} / \mathrm{m}^{2}, \quad \mu=1.89 \times 10^{10} \mathrm{~N} / \mathrm{m}^{2}, \quad a_{0}=0.61 \times 10^{-10} \mathrm{~N},
$$




$$
\begin{gathered}
\rho=2.19 \times 10^{3} \mathrm{~kg} / \mathrm{m}^{3}, \quad \beta_{1}=0.05 \times 10^{5} \mathrm{~N} / \mathrm{m}^{2} \mathrm{~K}, \quad \beta_{0}=0.05 \times 10^{5} \mathrm{~N} / \mathrm{m}^{2} \mathrm{~K}, \\
C^{*}=966 \mathrm{~J} /(\mathrm{kgk}), \quad K^{*}=252 \mathrm{~J} / \mathrm{msK}, \quad j_{0}=0.196 \times 10^{-4} \mathrm{~m}^{2}, \\
\lambda_{0}=\lambda_{1}=0.37 \times 10^{10} \mathrm{~N} / \mathrm{m}^{2}, \quad t_{0}=0.01, \quad t_{1}=0.0001, \quad T_{0}=293 \mathrm{~K} .
\end{gathered}
$$

The physical constants for elastic medium (granite) as [33]:

$\lambda^{e}=0.884 \times 10^{10} \mathrm{~N} / \mathrm{m}^{2}, \quad \mu^{e}=1.2667 \times 10^{10} \mathrm{~N} / \mathrm{m}^{2}, \quad \rho^{e}=2.6 \times 10^{3} \mathrm{Kg} / \mathrm{m}^{3}$.

The computations are carried out for the value of non-dimensional time $t=0.2$ in the range of $0 \leq y \leq 10$ and on the surface $x=1.0$. The numerical values for normal displacement, normal force stress, temperature distribution and microelongation are shown in Figs 2-5 for Green Lindsay (GL) theory $\delta_{1 k}=0, \delta_{2 k}=1$ and mechanical force with magnitude: $P_{1}=1.0, \omega=\omega_{0}+\iota \xi$, $\omega_{0}=-0.2, \xi=0.1$ and $b=0.8$.

(a) Thermoelastic microelongated solid (TMS) with $Q=1.0$ by solid line with dashed symbol

(b) Thermoelastic microelongated solid (TMS) with $Q=10.0$ by dashed line with centered symbol symbol

(c) Thermoelasic solid (TS) with $Q=1.0$ by dashed line with centered

(d) Thermoelasic solid (TS) with $Q=10.0$ by dotted line with centered symbol $\times$.

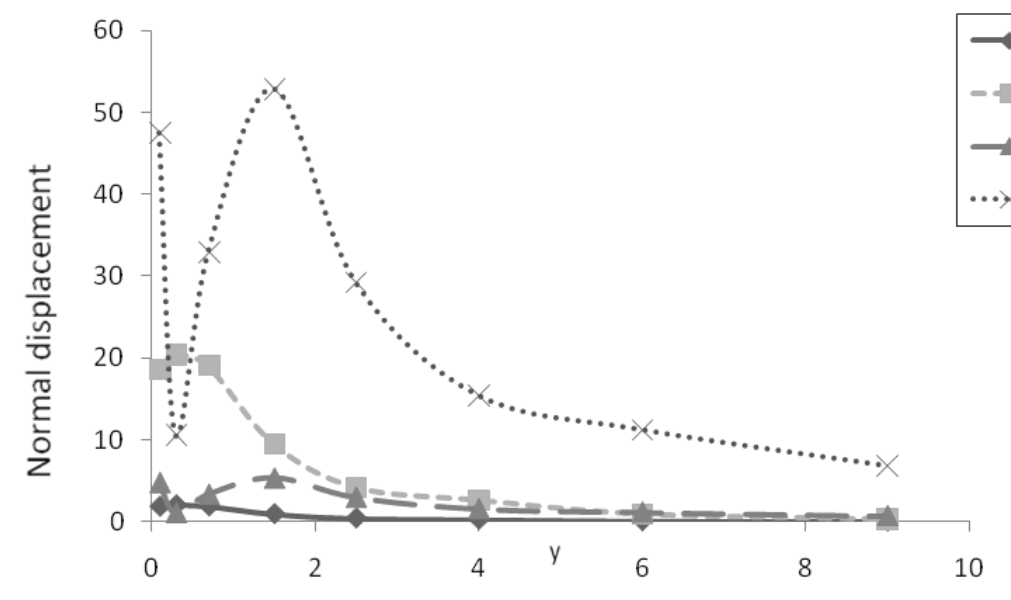

Fig. 2. Normal displacement variation with horizontal distance 


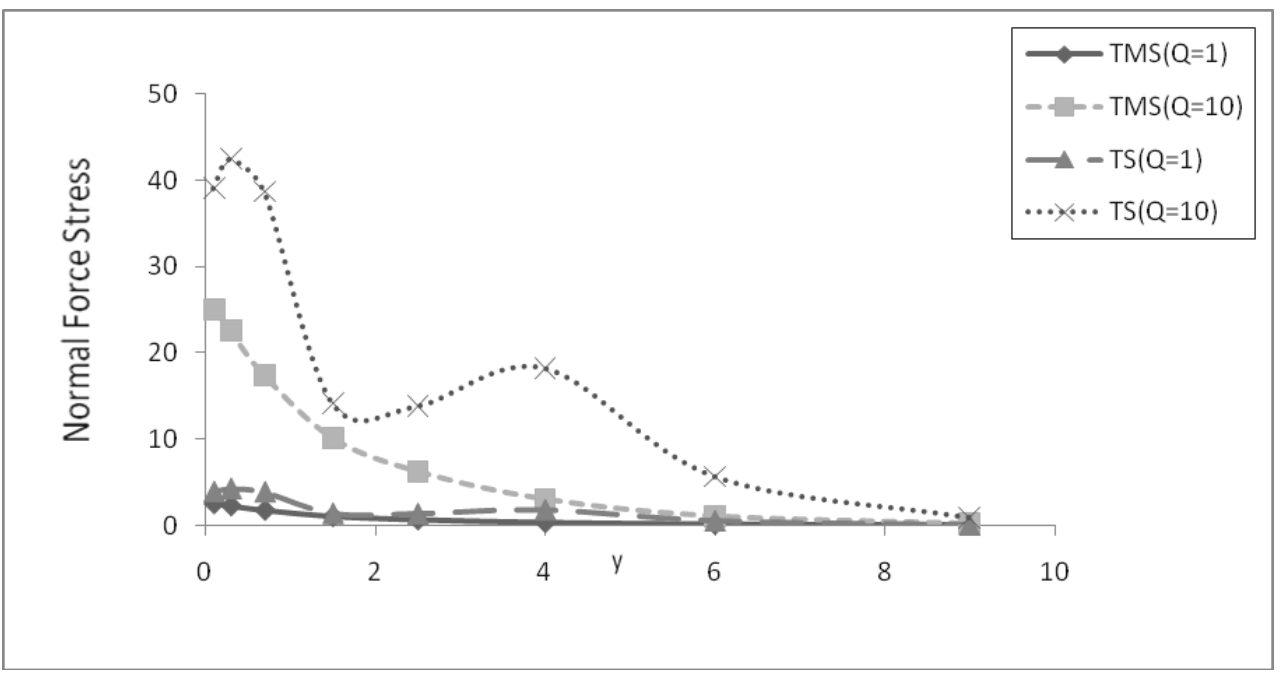

Fig. 3. Normal force stress variation with horizontal distance

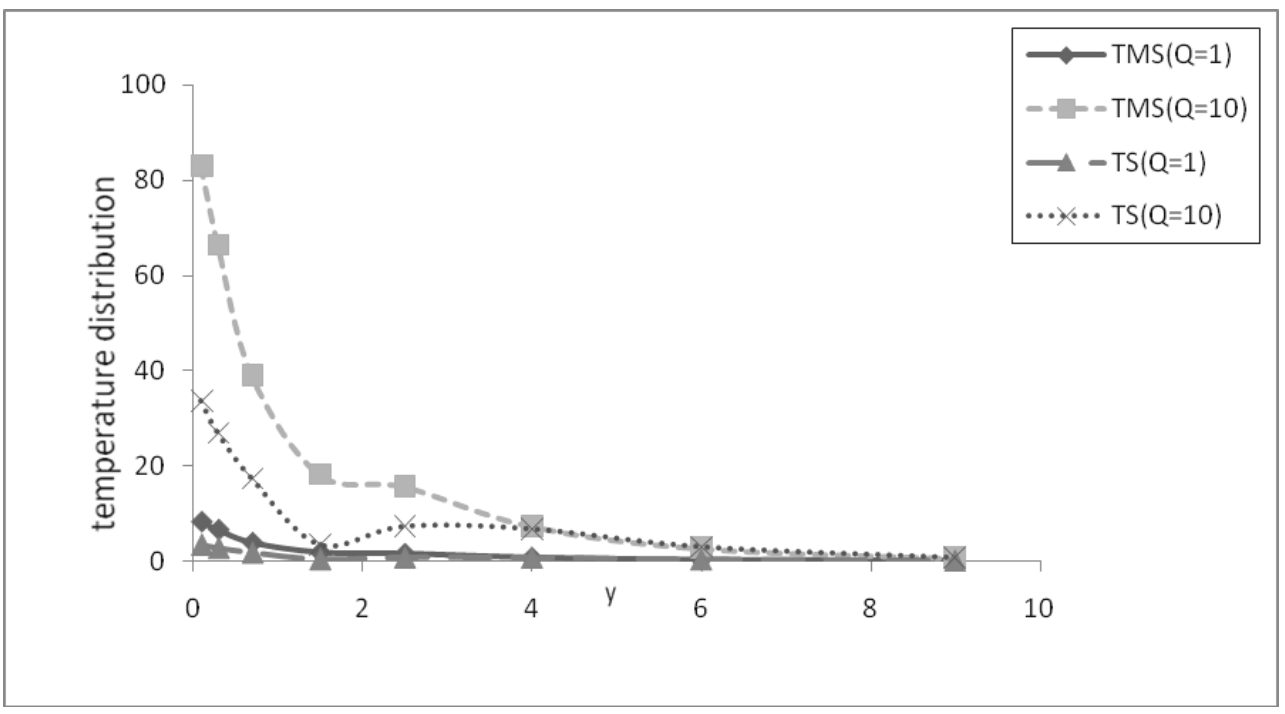

Fig. 4. Temperature distribution variation with horizontal distance

\section{Discussion}

As expected, the values of normal displacement, normal force stress, temperature distribution, and microelongation near the point of application of source are bigger, when the magnitude of internal heat source is larger 


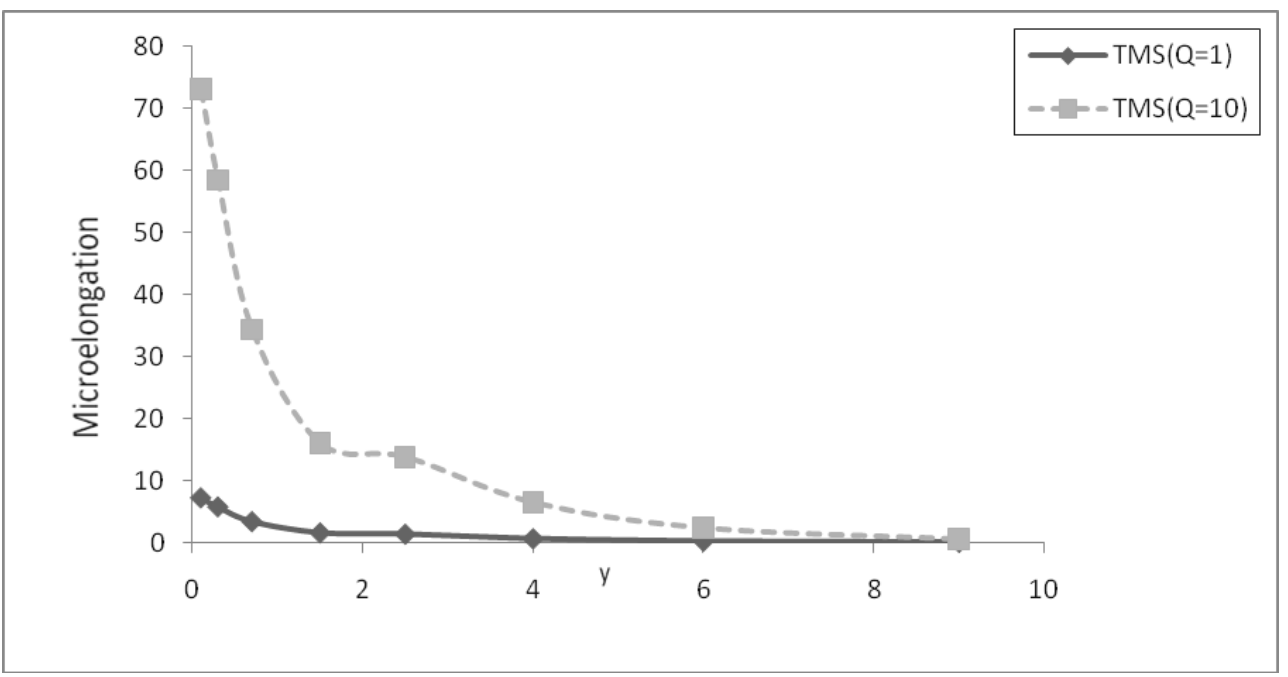

Fig. 5. Microelongation variation with horizontal distance

$(Q=10.0)$ as compared to that, when the magnitude of internal heat source is $(Q=1.0)$ in the range of $0 \leq y \leq 10.0$. From the Figs 2 and 3, it is very much clear that normal displacement and normal force stress vary in similar manner. The variation of all the quantities tends to zero as horizontal distance increases. These variations of normal displacement, temperature distribution, normal force stress and microelongation are shown in Figs $2-5$, respectively.

\section{Conclusion}

1. Microelongation and internal heat source have appreciable effect on all quantities.

2. The magnitude of quantities in the neighbourhood of mechanical force increases with increase in magnitude of internal heat source.

3. The problem may also be discussed in context of mechanical force (i.e. $Q \rightarrow 0$ ) and internal heat source (i.e. $P_{1} \rightarrow 0$ ), separately. The graphical results of these expressions may also be discussed in similar way.

4. The problem finds wide applications in dynamics and thermoelastic theory, including solid-liquid crystals, composite reinforced materials and porous media. 
Internal Heat Source in Thermoelastic Microelongated Solid ...

\begin{tabular}{l|l}
$\begin{array}{l}\text { List of used symbols } \\
\text { and notations }\end{array}$ & List of used abbreviations \\
\hline$\sigma=\sigma_{k k}$ & microelongational stress tensor \\
$s=s_{k k}$ & component of stress tensor \\
$\alpha_{t_{1}}$ and $\alpha_{t_{2}}$ & coefficient of linear thermal expansion, \\
$a_{0}, \lambda_{0}, \lambda_{1}$ & microelongational constants \\
$t_{0}, t_{1}$ & thermal relaxation times \\
$\rho$ & density of microelongated medium \\
$j_{0}$ & Microinertia \\
$K^{*}$ & coefficient of thermal conductivity \\
$C^{*}$ & specific heat at constant strain \\
$m_{k}$ & component of microstretch vector \\
$T$ & thermodynamic temperature above reference \\
& temperature $T_{0}$ \\
$\varphi$ & microelongational scalar \\
$\lambda, \mu$ & lame's constants \\
$\delta_{k l}$ & kronecker delta \\
$\vec{u}$ & displacement vector of microelongated solid \\
$Q$ & internal heat source \\
$\vec{u}^{e}$ & displacement vector of elastic solid \\
$\lambda^{e}, \quad \mu^{e}$ & elastic constants \\
$\rho^{e}$ & density of elastic solid \\
$\omega$ & complex frequency \\
$b$ & wave number in $y$-direction \\
&
\end{tabular}

\section{REFERENCES}

[1] Eringen, A. C., E. S. Suhubi. Nonlinear Theory of Simple Micro-elastic Solids I. International Journal of Engineering Science, 2 (1964), 189-203.

[2] Suhubi, E. S., A. C. ERIngen. Nonlinear Theory of Micro-elastic Solids II. International Journal of Engineering Science, 2 (1964), 389-404.

[3] ERIngen, A. C. Linear Theory of Micropolar Elasticity, ONR Techanical report No. 29, School of Aeronautics, Aeronautics and Engineering Science, Purdue University, 1965.

[4] ERIngen, A. C. A Unified Theory of Thermomechanical Materials. International Journal of Engineering Science, 4 (1966), 179-202.

[5] Eringen, A. C. Linear Theory of Micropolar Elasticity. Journal of Mathematics and Mechanics, 15 (1996), 909-923. 
[6] Eringen, A. C. Micropolar Elastic Solids with Strech. Ari Kitabevi Matbassi, 24 (1971), 1-18.

[7] Nowacki, W. Couple Stresses in the Theory of Thermoelasticity III. Bulletin of the Polish Academy of Sciences Techanical Sciences, 8 (1966), 801-809.

[8] Eringen, A. C. Foundation of Micropolar Thermoelasticity, Courses and Lectures, No. 23, CISM, Vienna and New York, Udine, Springer-Verlag, 1970.

[9] Tauchert, T. R., W. D. Claus Jr., T. Ariman. The Linear Theory of Micropolar Thermoelasticity. International Journal of Engineering Science, 6 (1968), 36-47.

[10] Tauchert, T. R. Thermal Stresses in Micropolar Elastic Solids. Acta Mechanica, 11 (1971), 155-169.

[11] Nowacki, W., W. Olszak. Micropolar Thermoelasticity, In W. Nowacki and Olszak (eds), Micropolar thermoelasticity, CISM Courses and Lecture No. 151, Udine, Vienna, Springer-Verlag, 1974.

[12] Dhaliwal, R. S., A. Singh. Micropolar Thermoelasticity, Chapter 5, In R. B. Hetnarski (ed.), Thermal Stresses II, Mechanical and Mathematical Methods, Vol. 2, North-Holland, Amsterdam, 1987.

[13] Eringen, A. C., C. B. Kafadar. In A. C. Eringen (ed.), Continum Physics, Vol. 4, New York, Academic Press, 1976.

[14] Lord, H. W., Y. Shulman. A Generalized Dynamical Theory of Thermoelasticity. Journal of the Mechanics and Physics of Solids, 15 (1967), 299-306.

[15] Muller, I. M. The Coldness, Universal Function in Thermoelastic Bodies. Rational Mechanics Analysis, 41 (1971), 319-332.

[16] Green, A. E., N. Laws. On the Entropy Production Inequality. Archives of Rational Mechanics and Analysis, 45 (1972), 45-47.

[17] Green, A. E., K. A. Lindsay. Thermoelasticity. Journal of Elasticity, 2 (1972), $1-7$.

[18] Suhubi, E. S. Thermoelastic Solids in Continuum Physics, New York, 1975.

[19] Barber, J. R. Thermoelastic Displacements and Stresses due to a Heat Source moving over the Surface of a Half Plane. ASME, Transactions, Journal of Applied Mechanics, 51 (1984), 636-640.

[20] Sherief, H. H. Fundamental Solution of the Generalized Thermoelastic Problem for Short Times. Journal of Thermal Stresses, 9 (1986), No. 2, 151-164.

[21] Dhaliwal, R. S., S. R. Majumdar, J. Wang. Thermoelastic Waves in an Infinite Solid caused by a Line Heat Source. International Journal of Mathematics and Mathematical Sciences, 20 (1997), No. 2, 323-334.

[22] Chandrasekharaiah, D. S., K. S. SRinath. Thermoelastic Interactions without Energy Dissipation due to a Point Heat Source. Journal of Elasticity, 50 (1998), 97-108.

[23] Sharma, J. N., R. S. Chauhan, R. Kumar. Time-harmonic Sources in a Generalized Thermoelastic Continuum. Journal of Thermal Stresses, 23 (2000), No. 7, 657-674. 
[24] Sharma, J. N., R. S. Chauhan. Mechanical and Thermal Sources in a Generalized Thermoelastic Half-space. Journal of Thermal Stresses, 24 (2001), No. 7, 651-675.

[25] Sarbani, C., C. Amitava. Transient Disturbance in a Relaxing Thermoelastic Half-space due to Moving Internal Heat Source. International Journal of Mathematics and Mathematical Sciences, 22 (2004), 595-602.

[26] Youssef, H. M. Generalized Thermoelastic Infinite Medium with Spherical Cavity subjected to Moving Heat Source. Computers Mathematical Modelling, 21 (2010), No. 2, 211-225.

[27] Shaw, S., B. Mukhopadhyay. Periodically Varying Heat Source Response in a Functionally Graded Microelongated Medium. Applied Mathematics and Computation, 128 (2012), No. 11, 6304-6313.

[28] Shaw, S., B. Mukhopadhyay. Moving Heat Source Response in a Thermoelastic Microelongated Solid. Journal of Engineering Physics and Thermophysics, 86 (2013), No. 3, 716-722.

[29] Eringen, A. C. Microcontinuum Field Theories, Vol. 1, Foundations and Solids, New York, Springer Verlag, 1999.

[30] KiRis, A., E. InAN. 3-D Vibration Analysis of the Rectangular Microdamaged Plates, in Proceedings 8th International Conference on Vibration Problems (ICOVP), India, 2007, 207-214.

[31] De Cicco, S., L. NAppa. On the Theory of Thermomicrostretch Elastic Solids. Journal of Thermal Stresses, 22 (1999), 565-580.

[32] Ewing, W. M., W. S. Jardetzky, F. Press. Elastic Waves in Layered Media, New York, McGraw Hill, 1957.

[33] Bullen, K. E. An Introduction to Theory of Seismology, Cambridge, Cambridge University Press, 1963.

[34] Ailawalia, P., S. K. Sachdeva. Plane Strain Deformation in Thermoelastic Microelongated Solid. Civil and Environmental Research, 7 (2015), No. 2, 92-98.

\section{Appendix 1}

$$
\begin{aligned}
& l_{1}=\frac{(\lambda+2 \mu)}{\rho c_{1}^{2}}, \\
& l_{2}=\frac{(\lambda+\mu)}{\rho c_{1}^{2}}, \\
& l_{3}=\frac{\mu}{\rho c_{1}^{2}}, \\
& l_{4}=\frac{\beta_{1} \lambda_{0} c_{1}^{2}}{a_{0} \omega^{*} \beta_{0}},
\end{aligned}
$$


80 Praveen Ailawalia, Sunil Kumar Sachdeva, Devinder Singh Pathania

$$
\begin{aligned}
& l_{5}=\frac{\lambda_{1} c_{1}^{2}}{a_{0} \omega^{*}}, \\
& l_{6}=\frac{\lambda_{0}^{2}}{\rho a_{0} \omega^{*}}, \\
& l_{7}=\frac{\rho j_{0} \omega^{*} c_{1}^{2}}{2 a_{0}}, \\
& l_{8}=\frac{\rho C^{*} c_{1}^{2}}{K^{*} \omega^{*}}, \\
& l_{9}=\frac{\beta_{0}^{2} T_{0}}{K^{*} \omega^{*} \rho}, \\
& l_{10}=\frac{\beta_{0} \beta_{1} T_{0} c_{1}^{2}}{K^{*} \omega^{*} \lambda_{0}}, \\
& l_{11}=\frac{\rho c_{1}^{4}}{K^{*} \omega^{*} T_{0}}, \\
& l_{12}=\frac{\lambda}{\rho c_{1}^{2}} .
\end{aligned}
$$

\section{Appendix 2}

$$
\begin{aligned}
D & \equiv \frac{d}{d x}, \\
B_{1} & =\omega^{2}+l_{3} b^{2}, \\
B_{2} & =\left(1+t_{1} \delta_{2 k} \omega\right), \\
B_{3} & =\omega^{2}+l_{1} b^{2}, \\
B_{4} & =b^{2}+l_{5}+l_{7} \omega^{2}, \\
B_{5} & =\left(1+t_{0} \delta_{1 k} \omega\right), \\
B_{6} & =\omega\left(1+t_{0} \delta_{1 k} \omega\right), \\
B_{7} & =b^{2}+l_{8} B_{5} \omega .
\end{aligned}
$$


Internal Heat Source in Thermoelastic Microelongated Solid ...

Appendix 3

$$
\begin{aligned}
A= & \frac{-1}{l_{1} l_{3}}\left[l_{1} l_{3}\left(B_{4}+B_{7}\right)-l_{1} B_{3}+l_{3} B_{1}+l_{3} l_{6}+B_{2} l_{3} l_{9} B_{6}+b^{2} l_{2}^{2}\right] \\
B= & \frac{-1}{l_{1} l_{3}}\left[-l_{1} B_{2} l_{4} l_{10} l_{3} \omega+l_{1} l_{3} B_{4} B_{7}+l_{1} B_{3}\left(B_{4}+B_{7}\right)-l_{1} b^{2} B_{2} B_{6} l_{9}+b^{2} l_{1} B_{6}\right. \\
& -B_{1} l_{3}\left(B_{4}+B_{7}\right)+B_{1} B_{3}-b^{2} l_{2}^{2}\left(B_{4}+B_{7}\right)+l_{3} l_{6} l_{10} B_{2} \omega-l_{3} l_{9} B_{2} B_{4} B_{6} \\
& \left.-l_{9} B_{2} B_{3} B_{6}-l_{3} l_{6} B_{7}-l_{3} l_{4} l_{9} B_{2} B_{6}-B_{3} l_{6}\right] \\
C= & \frac{-1}{l_{1} l_{3}}\left[B_{2} B_{3} l_{1} l_{4} l_{10} \omega+B_{3} B_{4} B_{7} l_{1}-b^{2} l_{1} l_{6} l_{10} B_{2} \omega+b^{2} l_{1} l_{9} B_{2} B_{4} B_{6}-b^{2} l_{1} l_{6} B_{7}\right. \\
& -b^{2} l_{1} l_{4} l_{9} B_{2} B_{6}+B_{1} B_{2} l_{3} l_{4} l_{10} \omega^{2}-l_{3} B_{1} B_{4} B_{7}+B_{1} B_{3}\left(B_{4}+B_{7}\right)+b^{2} B_{1} B_{2} B_{6} l_{9} \\
& +b^{2} l_{2}^{2} B_{2} l_{4} l_{10} \omega+b^{2} l_{2}^{2} B_{4} B_{7}-2 b^{2} B_{7} l_{2} l_{6}-2 b^{2} B_{2} B_{6} l_{2} l_{4} l_{9}-l_{6} l_{10} B_{2} B_{3} \omega \\
& \left.+B_{2} B_{3} B_{4} B_{6} l_{9}+B_{3} B_{7} l_{6}+B_{2} B_{3} B_{6} l_{4} l_{9}-b^{2} l_{6} B_{1}\right] \\
& \frac{-1}{l_{1} l_{3}}\left[-l_{4} l_{10} B_{1} B_{2} B_{3} \omega-B_{1} B_{3} B_{4} B_{7}+b^{2} l_{6} l_{10} B_{1} B_{2} \omega-b^{2} l_{9} B_{1} B_{2} B_{4} B_{6}\right. \\
& \left.+b^{2} l_{6} B_{1} B_{7}+b^{2} l_{4} l_{9} B_{1} B_{2} B_{6}\right] \\
R= & b^{2} l_{11} B_{1} B_{2} B_{4} B_{5}\left(l_{4}-B_{4}\right)
\end{aligned}
$$

\section{Appendix 4}

$$
\begin{aligned}
H_{1 n} & =\frac{i b\left[\left(l_{1}-l_{2}\right) k_{n}^{2}-B_{1}\right]}{\left[\left(B_{3}-b^{2} l_{2}\right) k_{n}-l_{3} k_{n}^{3}\right]}, \\
H_{2 n} & =\frac{\left[l_{3} k_{n}^{4}-\left(B_{4} l_{3}+B_{3}\right) k_{n}^{2}+\left(B_{3} B_{4}-b^{2} l_{6}\right)\right] H_{1 n}-i b\left[l_{2} k_{n}^{3}-\left(l_{2} B_{4}-l_{6}\right) k_{n}\right]}{i b\left[B_{2}\left(k_{n}^{2}-B_{4}\right)+B_{2} l_{4}\right]} \\
H_{3 n} & =\frac{\left(l_{1} k_{n}^{2}-B_{1}-i b l_{2} k_{n} H_{1 n}+B_{2} k_{n} H_{2 n}\right)}{k_{n}}, \\
H_{4 n} & =i b l_{12} H_{1 n}-B_{2} H_{2 n}+H_{3 n}-l_{1} k_{n}, \\
H_{5 n} & =l_{3}\left(i b-k_{n} H_{1 n}\right), \\
H_{6 n} & =i b l_{1} H_{1 n}-B_{2} H_{2 n}+H_{3 n}-l_{12} k_{n}, \\
S & =\frac{R Q^{*}}{E},
\end{aligned}
$$


82 Praveen Ailawalia, Sunil Kumar Sachdeva, Devinder Singh Pathania

$$
\begin{aligned}
& S_{1}=i b B_{1} S, \\
& S_{2}=i b B_{1}\left(B_{3} B_{4}-b^{2} l_{6}\right) S, \\
& S_{3}=B_{1} S, \\
& S_{4}=-\left(B_{2} S_{2}-S_{3}+i b l_{12} S_{1}\right), \\
& S_{5}=i b l_{3} S, \\
& S_{6}=-\left(B_{2} S_{2}-S_{3}+i b l_{1} S_{1}\right) .
\end{aligned}
$$

\section{Appendix 5}

$$
\begin{aligned}
G & =\frac{b^{2}\left(a_{1}^{2}+a_{3}^{2}-a_{2}^{2}\right)+\left(a_{1}+a_{3}\right) \omega^{2}}{a_{1} a_{3}}, \\
L & =\frac{b^{4} a_{1} a_{3}+b^{2} \omega^{2}\left(a_{1}+a_{3}\right)+\omega^{4}}{a_{1} a_{3}}, \\
a_{1} & =\frac{\lambda^{e}+2 \mu^{e}}{\rho^{e} c_{1}^{2}}, \\
a_{2} & =\frac{\lambda^{e}+\mu^{e}}{\rho^{e} c_{1}^{2}} \\
a_{3} & =\frac{\mu^{e}}{\rho^{e} c_{1}^{2}}, \\
L_{1 n} & =\frac{\omega^{2}+b^{2} a_{3}-a_{1} r_{n}^{2}}{i b a_{2} r_{n}}, \\
L_{2 n} & =\frac{\left(\lambda^{e}+2 \mu^{e}\right) r_{n}+i b \lambda^{e} L_{1 n}}{\rho c_{1}^{2}}, \\
L_{3 n} & =\frac{\lambda^{e} r_{n}+i b\left(\lambda^{e}+2 \mu^{e}\right) L_{1 n}}{\rho c_{1}^{2}}, \\
L_{4 n} & =\frac{\mu^{e}\left(i b+r_{n} L_{1 n}\right)}{\rho c_{1}^{2}} .
\end{aligned}
$$

